\title{
Secondary Nucleation by Interparticle Energies. II. Kinetics
}

\section{Journal Article}

Author(s):

Ahn, Byeongho (D); Bosetti, Luca (D); Mazzotti, Marco

Publication date:

2022-01-05

Permanent link:

https://doi.org/10.3929/ethz-b-000519706

\section{Rights / license:}

Creative Commons Attribution-NonCommercial-NoDerivatives 4.0 International

\section{Originally published in:}

Crystal Growth \& Design 22(1), https://doi.org/10.1021/acs.cgd.1c00928

\section{Funding acknowledgement:}

788607 - Studying Secondary Nucleation for the Intensification of Continuous Crystallization (EC) 


\title{
Secondary Nucleation by Interparticle Energies. II. Kinetics
}

\author{
Byeongho Ahn,* Luca Bosetti,* and Marco Mazzotti* \\ Cite This: https://doi.org/10.1021/acs.cgd.1c00928 \\ Read Online
}

ACCESS |

山ll Metrics \& More

Article Recommendations

Supporting Information

\begin{abstract}
This work presents a mathematical model that describes growth, homogeneous nucleation, and secondary nucleation that is caused by interparticle interactions between seed crystals and molecular clusters in suspension. The model is developed by incorporating the role of interparticle energies into a kinetic rate equation model, which yields the time evolution of nucleus and seed crystal populations, as in a population balance equation model, and additionally that of subcritical molecular clusters, thus revealing an important role of each population in crystallization. Seeded batch crystallization at a constant temperature has been simulated to demonstrate that the interparticle interactions increase the concentration of the critical clusters by several orders of magnitude, thus causing

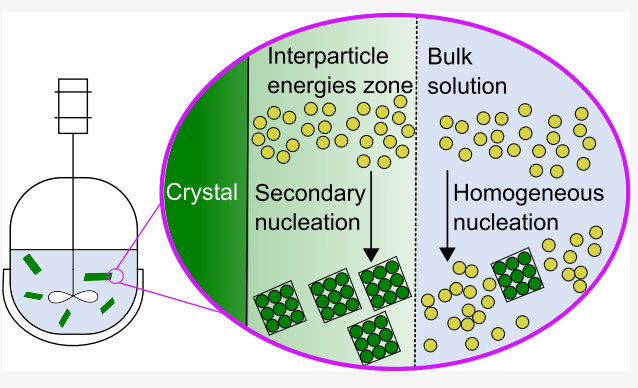
secondary nucleation. This explains how secondary nucleation can occur at a low supersaturation that is insufficient to trigger primary nucleation. Moreover, a sensitivity analysis has shown that the intensity of the interparticle energies has a major effect on secondary nucleation, while its effective distance has a minor effect. Finally, the simulation results are qualitatively compared with experimental observations in the literature, thus showing that the model can identify operating conditions at which primary or secondary nucleation is more prone to occur, which can be used as a useful tool for process design.
\end{abstract}

\section{INTRODUCTION}

Crystallization is an essential unit operation used as a purification technique in various industries for the production of chemicals, agrochemicals, nutritionals, and pharmaceuticals. Crystallization process is often initiated by the addition of seed crystals (seeds) of the target product in a supersaturated solution. While these seeds grow in the solution, they can generate new crystals (i.e., nuclei), through the phenomenon called secondary nucleation, which is widespread in industrial crystallization, ${ }^{1}$ in particular, in the continuous crystallization of active pharmaceutical ingredients. ${ }^{2}$ Hence, to operate and design continuous crystallization efficiently, we need a sound understanding of secondary nucleation mechanisms.

Traditionally, secondary nucleation has been largely attributed to microbreakages of seed crystals caused by crystalimpeller collisions, ${ }^{3}$ that is, attrition. ${ }^{1,4,5}$ However, recent research has noted that this mechanism cannot describe secondary nucleation completely, ${ }^{3,6-13}$ based on two main reasons. First, secondary nucleation can occur even when the crystal microbreakages are prevented by precluding any crystalimpeller collision. ${ }^{11-13}$ Second, the attrition mechanism cannot explain some crucial experimental observations according to which secondary nucleation can produce nuclei having a polymorphic $^{14}$ or chiral $^{15-17}$ form different from that of the seeds. In contrast with these observations, secondary nuclei generated by the attrition mechanism must exhibit the same polymorphic form and the same handedness in the case of chiral molecules of the seeds because these nuclei are the broken pieces of the seeds. These reasons clearly highlight that when describing secondary nucleation, we need to consider not only the attrition mechanism but also other secondary nucleation mechanisms.

Another perspective for rationalizing secondary nucleation suggests that secondary nucleation is induced by interparticle interactions, ${ }^{18}$ in particular, the interaction energies between seed crystals and nearby molecular clusters. From this perspective, the interactions facilitate nucleation by reducing the energy barrier for nucleation in the vicinity of the seeds. This type of secondary nucleation can be called secondary nucleation caused by interparticle energies (SNIPE). As opposed to secondary nucleation by attrition, the SNIPE mechanism can explain why some secondary nuclei exhibit a polymorphic/chiral crystal structure different from that of the seeds; this is because the interparticle energies decrease the energy barrier for nucleation independent of the crystal structure of the relevant cluster, ${ }^{15,18}$ thus causing secondary nucleation of any crystal structures.

A simple version of the SNIPE mechanism is the embryocoagulation secondary nucleation (ECSN), ${ }^{18}$ a key mechanism according to recent reviews on secondary nucleation. ${ }^{1,4,5}$ However, despite its important contribution to our under-

Received: August 13, 2021

Revised: October 19, 2021 
standing of the SNIPE mechanism, the ECSN model has three critical limitations. First, the ECSN model assumes that subcritical molecular clusters are monodispersed. This assumption contradicts the classical nucleation theory ${ }^{19}$ (CNT) whereby a cluster population develops in solution following a Boltzmann distribution. Second, the ECSN model further assumes that the subcritical molecular clusters grow by aggregation only, thus being inconsistent with the CNT where the clusters grow via a sequence of molecule attachments. Third, the ECSN model cannot describe the temporal evolution of any of the three key populations in crystallization-the (subcritical) molecular clusters, nuclei, and seed crystals - thus limiting a deeper understanding of how the SNIPE mechanism works.

The goal of this work is to develop a mathematical model that simultaneously describes homogeneous nucleation, growth, and the SNIPE phenomenon while overcoming the three limitations of the ECSN model. To this aim, the present work extends the conventional kinetic rate equation (KRE) model of nucleation by including a description of the SNIPE mechanism. The structure of this article is as follows. In Section 2, we provide an overview of the conventional KRE model that describes homogeneous nucleation and growth while being consistent with the CNT. In Section 3, we incorporate the description of the SNIPE mechanism into the conventional KRE model. In Section 4, using the developed model, we demonstrate how the SNIPE mechanism works, show the results of a sensitivity analysis, and compare the simulation results qualitatively with available experimental observations.

\section{CONVENTIONAL KRE MODEL OF NUCLEATION}

To describe the SNIPE mechanism, we extend the conventional KRE model of nucleation (i.e., the Szilard model ${ }^{19,20}$ ) by integrating the effect of the interparticle energies. Hence, knowing the working principle of the Szilard model is essential for understanding how our SNIPE model works. To give a brief overview, the basic principle of the model and its governing equations are presented in this section, followed by equations for the attachment and detachment rate constants that are necessary for solving the governing equations.

2.1. Principle. The Szilard model is a well-established theoretical framework that describes homogeneous nucleation and growth via a series of molecule attachments to and detachments from the molecular clusters, ${ }^{19,21}$ that is, a description consistent with the CNT. ${ }^{19}$ In this approach, a molecular cluster consisting of $n$ solute molecules (i.e., a $n$-sized cluster) represents a solid particle (i.e., a crystal), when its size $n$ is larger than the critical nucleus size $n^{*}$ (i.e., $n>n^{*}$ ) defined in the CNT. ${ }^{19}$ Otherwise (i.e., when $n \leq n^{*}$ ), the $n$-sized cluster is considered as part of the liquid phase; hence, its volume is excluded from the calculation of the solid-phase volume as in the literature. ${ }^{19}$ The $n$-sized clusters can grow to $(n+1)$-sized clusters via molecule attachments, while $(n+1)$-sized clusters decay to the $n$-sized clusters through molecule detachments. This mechanism can be viewed as the following pseudo reaction $^{19,21}$

$$
W_{n}+W_{1} \underset{k_{n+1}^{\mathrm{d}}}{\stackrel{k_{n}^{\mathrm{a}}}{\rightleftarrows}} W_{n+1}
$$

where $W_{n}$ represents a $n$-sized cluster, $k_{n}^{\mathrm{a}}$ is the rate constant of monomer attachment to the $n$-sized clusters, and $k_{n+1}^{\mathrm{d}}$ is the rate constant of monomer detachment from the $(n+1)$-sized clusters. This formula suggests that the temporal evolution of the cluster concentrations can be described using rate equations once the rate constants $k_{n}^{\mathrm{a}}$ and $k_{n+1}^{\mathrm{d}}$ are known.

2.2. Governing Equations. On the basis of eq 1, we can formulate a system of KREs for a closed system, each characterizing the rate at which the concentration of the $n$ sized clusters evolves upon molecule attachments and detachments: ${ }^{19}$

$$
\begin{aligned}
\frac{\mathrm{d} Z_{n}}{\mathrm{~d} t} & =k_{n-1}^{\mathrm{a}} Z_{1} Z_{n-1}+k_{n+1}^{\mathrm{d}} Z_{n+1}-k_{n}^{\mathrm{a}} Z_{1} Z_{n}-k_{n}^{\mathrm{d}} Z_{n} \\
(n & \left.=2,3, \ldots, n_{\max }\right) \\
\frac{\mathrm{d} Z_{1}}{\mathrm{~d} t} & =-\sum_{n=2}^{n_{\max }} n \frac{\mathrm{d} Z_{n}}{\mathrm{~d} t}
\end{aligned}
$$

where $Z_{n}(t)$ is the concentration of the $n$-sized clusters (i.e., their number per unit suspension volume) and $Z_{n_{\max }+1}(t) \equiv 0$, with $n_{\max }$ being the upper bound of the cluster size, which must be sufficiently large to avoid any effect on the numerical solution. ${ }^{22}$ Equation $2 \mathrm{~b}$ is the mass balance for the solute, ensuring that the total number of solute molecules is conserved. The initial condition is as follows

$$
Z_{n}(0)=Z_{n, 0}
$$

where $Z_{n, 0}$ is the initial cluster size distribution. The system of eqs $2 \mathrm{a}$ and $2 \mathrm{~b}$ can be solved with the initial condition (eq 3 ) to obtain the temporal evolution of any initial cluster size distribution for a given set of rate constants (possibly evolving in time, for instance, due to temperature changes).

2.3. Attachment and Detachment Rate Constants. The attachment rate constant $k_{n}^{\mathrm{a}}$ is determined by the rate-limiting mechanism of the monomer transport to the clusters. It is common that the monomer transfer is limited by the monomer integration step on a cluster surface, for example, in the crystallization of L-glutamic acid in an aqueous solution, ${ }^{23}$ lactose in an aqueous solution, ${ }^{24}$ and paracetamol in an ethanol solution. ${ }^{25}$ Under this surface-integration limited mechanism, $k_{n}^{\text {a }}$ is given by ${ }^{19,26}$

$$
k_{n}^{\mathrm{a}}=\alpha k_{\mathrm{s}}\left(V_{1} / k_{\mathrm{v}}\right)^{1 / 3} \mathrm{Dn}^{2 / 3}
$$

where $\alpha$ is the sticking coefficient, $k_{\mathrm{s}}$ is the surface area shape factor (e.g., $k_{\mathrm{s}}=\pi$ for a sphere), $k_{\mathrm{v}}$ is the volume shape factor (e.g., $k_{\mathrm{v}}=\pi / 6$ for a sphere), $V_{1}$ is the volume of a solute molecule, and $D$ is the diffusion coefficient of the solute molecule in the solution. Equation 4 shows that $k_{n}^{\mathrm{a}}$ is a purely kinetic quantity, ${ }^{19}$ as it does not contain any thermodynamic parameter. The detachment rate constant $k_{n}^{\mathrm{d}}$ can be derived based on thermodynamic considerations about the concentration of clusters. ${ }^{19}$ For instance, at equilibrium, the monomer attachments to the $n$-sized clusters occur with the same frequency as the monomer detachments from $(n+1)$-sized clusters, thus kinetically ensuring the microscopic balance of the pseudo reaction (eq 1); this implies

$$
k_{n}^{\mathrm{a}} C_{1} C_{n}=k_{n+1}^{\mathrm{d}} C_{n+1}
$$

where $C_{n}$ is the equilibrium concentration of $n$-sized clusters at the actual level of $\Delta \mu$, that is, the thermodynamic driving force for crystallization; ${ }^{19}$ therefore, we write $C_{n}(\Delta \mu)$. The equilibrium cluster concentration $C_{n}$ can be expressed as ${ }^{19}$ 


$$
C_{n}=C_{0} \exp \left(-\frac{\Delta G_{n}}{k_{\mathrm{B}} T}\right)
$$

where $C_{0}$ is the concentration of nucleation sites in the system, $\Delta G_{n}(\Delta \mu)$ is the Gibbs free energy for the formation of a $n$-sized cluster from $n$ monomers at a given driving force $\Delta \mu, k_{\mathrm{B}}$ is Boltzmann constant, and $T$ is the absolute temperature of the system. The nucleation site concentration $C_{0}$ is typically defined as $V_{1}^{-1}$ in the literature, ${ }^{19,26}$ but a different value of $C_{0}$ calculated from a mass balance was used in this work to consider the bulk solubility $c_{\mathrm{e}}$ of a system, as explained in Appendix A. It is worth highlighting that $C_{0}$ is cancelled out in the nondimensional Szilard model (see eqs A.5a-A.8 with $E_{\text {st }}=1$ ), so the general behavior of the model is not affected by the choice of a specific value of $C_{0}$. We further note that the probability $C_{n} / C_{0}$ of finding a $n$-sized cluster at a given nucleation site is exponentially proportional to $-\Delta G_{n} / k_{\mathrm{B}} T,{ }^{19}$ thus indicating that the equilibrium cluster size distribution $C_{n}$ is a Boltzmann-type distribution. The driving force $\Delta \mu$ is defined as ${ }^{23}$

$$
\Delta \mu=k_{\mathrm{B}} T \ln \left(\frac{Z_{1}}{C_{1, \mathrm{e}}}\right)=k_{\mathrm{B}} T \ln s
$$

where $C_{1, \mathrm{e}} \equiv C_{1}(0)$ is the monomer concentration at phase equilibrium $(\Delta \mu=0)$ and $s=Z_{1} / C_{1, \mathrm{e}}$ is the supersaturation (ratio) based on the actual monomer concentrations. According to the CNT, $\Delta G_{n}$ is given by ${ }^{19}$

$$
\Delta G_{n}=-n \Delta \mu+\gamma b n^{2 / 3}
$$

where $\gamma$ is the specific surface energy of a cluster and $b=k_{s}\left(V_{1} /\right.$ $\left.k_{\mathrm{v}}\right)^{2 / 3}$ is the surface area of a solute molecule. Combining eqs 5 and 6 , with a consideration that $C_{1}$ is very close or equal to $Z_{1}{ }^{19}$ leads to

$$
k_{n+1}^{\mathrm{d}}=k_{n}^{\mathrm{a}} Z_{1} \exp \left(\frac{\Delta G_{n+1}-\Delta G_{n}}{k_{\mathrm{B}} T}\right)
$$

or with the help of eqs 7 and 8

$$
k_{n+1}^{\mathrm{d}}=k_{n}^{\mathrm{a}} C_{1, \mathrm{e}} \exp \left(\frac{\gamma b}{k_{\mathrm{B}} T}\left[(n+1)^{2 / 3}-n^{2 / 3}\right]\right)
$$

where $k_{0}^{\mathrm{a}}=0$ and $k_{1}^{\mathrm{d}}=0$ by definition. ${ }^{19}$ Note that eq 9 shows that $k^{\mathrm{d}}$ is determined by both kinetics (i.e., $k^{\mathrm{a}}$ ) and thermodynamics (i.e., $\Delta G) .{ }^{19}$

\section{MODELING SNIPE}

In this section, we extend the Szilard model to describe the SNIPE mechanism by incorporating the assumption that interparticle interactions stabilize cluster formation. This stabilization effect alters the thermodynamics of nucleation (i.e., $\Delta G$ ) near seed crystals such that the effective detachment rate constants $k^{\mathrm{d} \text {,eff }}(t)$ become smaller than those without stabilization, thus accelerating nucleation. For implementation, the identical governing equations of the Szilard model (eqs $2 \mathrm{a}$ and $2 \mathrm{~b}$ ) can be reused after replacing the original detachment rate constants $k^{\mathrm{d}}$ with $k^{\mathrm{d} \text {,eff }}$ whose formulation is described in the following by considering that only part of a cluster population close to seed crystals gets stabilized by the interparticle energies.

3.1. Interparticle Energies. In part I of this series, ${ }^{27}$ the thermodynamic aspects of the SNIPE mechanism are examined in detail by considering two classical interparticle forces, that is, van der Waals forces and Born repulsion forces. This contribution, as part II of this series, examines the kinetic aspect of the SNIPE mechanism by including the role of these interparticle interactions in the Szilard model under the following simplifying assumptions:

- As illustrated in Scheme 1a, a seed crystal is surrounded by a stabilization volume $V_{\text {st }}(t)$ consisting of a thin shell with

Scheme 1. Schematic representation of a seed crystal in a solution when a crystal is spherically shaped; not drawn to scale $^{a}$

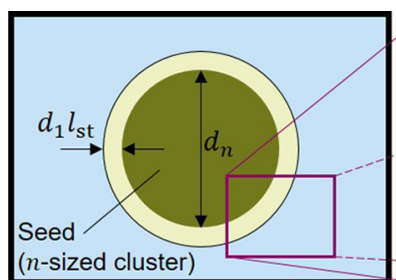

(a)

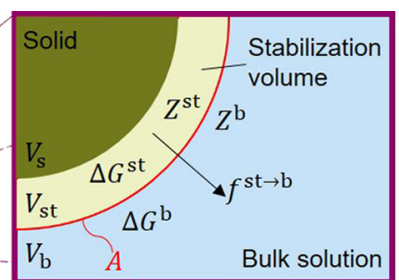

(b) $a^{a}$ (a) A seed crystal consisting of $n$ solute molecules is surrounded by the stabilization volume, consisting of a thin shell with thickness $d_{1} l_{\mathrm{st}}$, where $d_{1}$ is the characteristic length of a solute molecule and $l_{\text {st }}$ is an adjustable parameter. (b) A suspension volume consists of a solid phase volume $V_{s}$, a stabilization volume $V_{s t}$ and a bulk solution volume $V_{\mathrm{b}}$. Cluster formation occurring in $V_{\mathrm{b}}$ is governed by the Gibbs free energy $\Delta G^{\mathrm{b}}$, whereas the cluster formation in $V_{\text {st }}$ is controlled by the Gibbs free energy $\Delta G^{\text {st }}$ in that volume. The concentrations $Z^{\mathrm{b}}$ and $Z^{\mathrm{st}}$ represent the cluster concentrations in $V_{\mathrm{b}}$ and $V_{\mathrm{st}}$, respectively. A flux of molecular clusters $f^{\mathrm{st} \rightarrow \mathrm{b}}$ from $V_{\mathrm{st}}$ to $V_{\mathrm{b}}$ through the interfacial area $A$ is represented by an arrow.

thickness $d_{1} l_{\mathrm{st}}$, where $d_{1}$ is the characteristic length of a solute molecule and $l_{\mathrm{st}}(\geq 0)$ is an adjustable nondimensional parameter.

- A uniform field of interparticle energies is applied within $V_{\text {st }}$, which vanishes outside $V_{\text {st }}$; this external field stabilizes the cluster formation by decreasing $\Delta G_{n}$.

Under these assumptions, we can define the Gibbs free energy $\Delta G_{n}^{\text {st }}(\Delta \mu)$ for the formation of a $n$-sized cluster in $V_{\text {st }}$ at a given $\Delta \mu$ as follows (see also eq 11 in part I of this series)

$$
\Delta G_{n}^{\text {st }}=-n\left(\Delta \mu+k_{\mathrm{B}} T \ln E_{\mathrm{st}}\right)+\gamma b n^{2 / 3}
$$

where $E_{\mathrm{st}}(\geq 1)$ is a nondimensional parameter that accounts for the intensity of the interparticle energies. By substituting $\Delta G$ in eq 9 with $\Delta G^{\text {st }}$, the detachment rate constant $k_{n}^{\text {d,st }}$ in $V_{\text {st }}$ can be written as

$$
k_{n}^{\mathrm{d}, \mathrm{st}}=\frac{k_{n}^{\mathrm{d}, \mathrm{b}}}{E_{\mathrm{st}}}
$$

Here, $k_{n}^{\mathrm{d}, \mathrm{b}}\left(=k_{n}^{\mathrm{d}}\right)$ is the detachment rate constant in the bulk solution volume $V_{\mathrm{b}}(t)$, with the corresponding Gibbs free energy $\Delta G_{n}^{\mathrm{b}}\left(=\Delta G_{n}\right)$. Equation 12 shows that when the interparticle energies are present (i.e., $E_{\mathrm{st}}>1$ ), $k_{n}^{\mathrm{d}, \mathrm{st}}$ is smaller than $k_{n}^{\mathrm{d}, \mathrm{b}}$, thus implying that clusters grow faster in $V_{\text {st }}$ than they do in $V_{\mathrm{b}}$. Obviously, when the interparticle energies are absent (i.e., $E_{\mathrm{st}}=$ 1), $k_{n}^{\mathrm{d}, \mathrm{st}}$ is equivalent to $k_{n}^{\mathrm{d}}$, thus yielding no stabilization effect. To consider different kinetics in $V_{\mathrm{b}}$ and $V_{\text {st }}$ and to describe the evolution of an entire cluster population, the system can be divided into multiple compartments, each described by a mass balance with a corresponding kinetic description. In this work, the system/suspension volume $V_{\text {susp }}$ is partitioned into three compartments, as illustrated in Scheme $1 \mathrm{~b}$ : the bulk solution 
volume $V_{\mathrm{b}}$, the stabilization volume $V_{\mathrm{st}}$, and the volume of the solid phase $V_{s}$, with corresponding time-dependent volume fractions defined as

$$
\phi_{\mathrm{b}}=\frac{V_{\mathrm{b}}}{V_{\text {susp }}} \quad \phi_{\mathrm{st}}=\frac{V_{\mathrm{st}}}{V_{\text {susp }}} \quad \phi_{\mathrm{s}}=\frac{V_{\mathrm{s}}}{V_{\text {susp }}}
$$

with $\phi_{\mathrm{b}}(t)+\phi_{\mathrm{st}}(t)+\phi_{\mathrm{s}}(t)=1$. The solid volume fraction $\phi_{\mathrm{s}}$ can be calculated by considering that the solid phase consists of supercritical clusters ${ }^{19}$

$$
\phi_{\mathrm{s}}=V_{1} \sum_{n=n^{*}+1}^{n_{\max }} n Z_{n}
$$

where $V_{1} n$ approximates the volume of a $n$-sized cluster and $n^{*}=$ $(2 \gamma b / 3 \Delta \mu)^{3}$ is the critical size. ${ }^{19}$ Likewise, $\phi_{\text {st }}$ can be calculated by considering all stabilization (shell) volumes in the suspension

$$
\begin{aligned}
& \phi_{\mathrm{st}}=k_{\mathrm{v}} \sum_{n=n_{\mathrm{st}}}^{n_{\max }}\left[\left(d_{n}+2 l_{\mathrm{st}} d_{1}\right)^{3}-d_{n}^{3}\right] Z_{n} \\
& \approx 6 V_{1} l_{\mathrm{st}} \sum_{n=n_{\mathrm{st}}}^{n_{\max }} n^{2 / 3} Z_{n}
\end{aligned}
$$

where $n_{\text {st }}$ is the minimum cluster size that enables the existence of the stabilization volume and $d_{n}$ is the characteristic length of a $n$-sized cluster.

For simplicity, $n_{\mathrm{st}}$ is set to be the minimum size of the initial seed crystals, being on the order of $10^{13}$ in the present work (corresponding to $25-50 \mu \mathrm{m}$ for small organic molecules). The approximation in eq $15 \mathrm{~b}$ is valid, provided that the size of seed crystals (i.e., $d_{n}=d_{1} n^{1 / 3}$ ) is much larger than the thickness of the stabilization volume $d_{1} l_{\mathrm{st}}$, that is, $n^{1 / 3} \gg l_{\mathrm{st}}$, where $l_{\mathrm{st}}$ is varied from 1 to 100 in this study. Note that eq $15 \mathrm{~b}$ shows that $\phi_{\text {st }}$ is linearly proportional to the parameter $l_{\mathrm{st}}$ and the total surface area of seed crystals. The concentrations of $n$-sized clusters in $V_{\mathrm{b}}$ and $V_{\text {st }}$, denoted by $Z^{\mathrm{b}}$ and $Z^{\text {st }}$, respectively, can be determined by considering that the total number of $n$-sized clusters is conserved, that is,

$$
V_{\text {susp }} Z_{n}=V_{\mathrm{b}} Z_{n}^{\mathrm{b}}+V_{\mathrm{st}} Z_{n}^{\mathrm{st}}
$$

and by assuming that their ratio $Z_{n}^{\mathrm{b}} / Z_{n}^{\text {st }}$ is well approximated by that at equilibrium, $C_{n}^{\mathrm{b}} / C_{n}^{\text {st }}$, given by

$$
\frac{Z_{n}^{\mathrm{b}}}{Z_{n}^{\mathrm{st}}} \approx \frac{C_{n}^{\mathrm{b}}}{C_{n}^{\mathrm{st}}}=\frac{C_{0} \exp \left(-\Delta G_{n}^{\mathrm{b}} / k_{\mathrm{B}} T\right)}{C_{0} \exp \left(-\Delta G_{n}^{\mathrm{st}} / k_{\mathrm{B}} T\right)}
$$

where $C_{n}^{\text {st }}(\Delta \mu)$ and $C_{n}^{\mathrm{b}}(\Delta \mu)$, likewise in eq 6 , are the equilibrium concentrations of the $n$-sized clusters in $V_{\text {st }}$ and $V_{\mathrm{b}}$, respectively. Combining eqs $8,11,13,16$, and 17 yields

$$
\begin{aligned}
& Z_{n}^{\mathrm{b}}=\frac{1}{\phi_{\mathrm{st}} E_{\mathrm{st}}^{n}+\phi_{\mathrm{b}}} Z_{n} \\
& Z_{n}^{\mathrm{st}}=\frac{E_{\mathrm{st}}^{n}}{\phi_{\mathrm{st}} E_{\mathrm{st}}^{n}+\phi_{\mathrm{b}}} Z_{n}
\end{aligned}
$$

The mass balances for $Z^{\mathrm{b}}$ and $Z^{\text {st }}$ can be written as

$$
\begin{aligned}
\frac{\mathrm{d}}{\mathrm{d} t}\left(V_{\mathrm{b}} Z_{n}^{\mathrm{b}}\right)= & V_{\mathrm{b}}\left(k_{n-1}^{\mathrm{a}} Z_{1} Z_{n-1}^{\mathrm{b}}-k_{n}^{\mathrm{a}} Z_{1} Z_{n}^{\mathrm{b}}+k_{n+1}^{\mathrm{d}, \mathrm{b}} Z_{n+1}^{\mathrm{b}}\right. \\
& \left.-k_{n}^{\mathrm{d}, \mathrm{b}} Z_{n}^{\mathrm{b}}\right)+A f_{n}^{\mathrm{st} \rightarrow \mathrm{b}}
\end{aligned}
$$

$$
\begin{aligned}
\frac{\mathrm{d}}{\mathrm{d} t}\left(V_{\mathrm{st}} Z_{n}^{\mathrm{st}}\right)= & V_{\mathrm{st}}\left(k_{n-1}^{\mathrm{a}} Z_{1} Z_{n-1}^{\mathrm{st}}-k_{n}^{\mathrm{a}} Z_{1} Z_{n}^{\mathrm{st}}+k_{n+1}^{\mathrm{d}, \mathrm{st}} Z_{n+1}^{\mathrm{st}}\right. \\
& \left.-k_{n}^{\mathrm{d}, \mathrm{st}} Z_{n}^{\mathrm{st}}\right)-A f_{n}^{\mathrm{st} \rightarrow \mathrm{b}}
\end{aligned}
$$

where $f_{n}^{\mathrm{st} \rightarrow \mathrm{b}}$ is the flux of the $n$-sized clusters from $V_{\text {st }}$ to $V_{\mathrm{b}}$ through the interfacial area $A$, illustrated by an arrow in Scheme $1 \mathrm{~b}$, and the monomer concentration in each volume is approximated by $Z_{1}$, as in part I of this series. ${ }^{27}$ Summing up these two equations and using eqs $12,13,16,18$, and 19 lead to a system of governing equations for $Z_{n}\left(n=2,3, \ldots, n_{\max }\right)$, analogous to eq $2 \mathrm{a}$, that can be cast as follows

$$
\frac{\mathrm{d} Z_{n}}{\mathrm{~d} t}=k_{n-1}^{\mathrm{a}} Z_{1} Z_{n-1}-k_{n}^{\mathrm{a}} Z_{1} Z_{n}+k_{n+1}^{\mathrm{d}, \text { eff }} Z_{n+1}-k_{n}^{\mathrm{d}, \text { eff }} Z_{n}
$$

where the effective detachment rate constant $k_{n}^{\mathrm{d}, \text { eff }}$ is

$$
k_{n}^{\mathrm{d}, \mathrm{eff}}=\left(\frac{\phi_{\mathrm{st}} E_{\mathrm{st}}^{n-1}+\phi_{\mathrm{b}}}{\phi_{\mathrm{st}} E_{\mathrm{st}}^{n}+\phi_{\mathrm{b}}}\right) k_{n}^{\mathrm{d}}
$$

Equation 23 implicitly assumes that all clusters including the supercritical $\left(n>n^{*}\right)$ clusters experience the stabilization effect; it has been used in all simulations reported in this paper. This is because the influence of $k_{n>n^{*}}^{\text {d,eff }}$ on the simulation results $Z_{n}(t)$ is negligible compared to that of $k_{n \leq n^{*}}^{\mathrm{d} \text {,eff }}$ and setting $k_{n>n^{*}}^{\mathrm{d} \text {,eff }} \approx k_{n>n^{*}}^{\mathrm{d}}$ increases the computational time by a factor of 20 . Summarizing, a system of the governing equations (eqs $2 b$ and 22) can be solved with the initial condition (eq 3) for simulating a crystallization process in the presence of the stabilization effect that is described by eqs $4,10,13-15$, and 23 . The developed model contains 11 parameters: $\alpha_{\mathrm{s}}, k_{\mathrm{s}}, k_{\mathrm{v}}, V_{1}, D, T, \gamma, C_{1, \mathrm{e}}\left(\right.$ or $\left.c_{\mathrm{e}}\right)$, $Z_{n, 0}, E_{\mathrm{st}}$ and $l_{\mathrm{st}}$. As elaborated in Appendix B, the model can be made dimensionless by introducing two dimensionless variables

$$
\begin{aligned}
& Y_{n}=\frac{Z_{n}}{C_{1, \mathrm{e}}} \\
& \tau=\frac{t}{\left(k_{1}^{\mathrm{a}} C_{1, \mathrm{e}}\right)^{-1}}
\end{aligned}
$$

where $Y_{n}$ and $\tau$ are the nondimensional concentration and time, respectively. Consequently, the nondimensional model contains five dimensionless parameters: $\beta, \Omega, Y_{n, 0}=Z_{n, 0} / C_{1, \mathrm{e}}, E_{\mathrm{st}}$ and $l_{\mathrm{st}}$. Here, $\beta$ represents the volume fraction of monomers at phase equilibrium, defined as

$$
\beta=V_{1} C_{1, \mathrm{e}}
$$

and $\Omega$ is the dimensionless surface energy, defined as

$$
\Omega=\frac{\gamma b}{k_{\mathrm{B}} T}
$$

The nondimensional model has been solved numerically as explained in Appendix C.

\section{RESULTS}

To demonstrate the SNIPE mechanism and the application of the developed model to experimental observations in the literature, seeded batch crystallization processes have been simulated at a constant temperature with varying initial supersaturation, initial seed size distribution, strength, and effective range of the stabilization effect. For simulating such conditions, two parameters $(\Omega$ and $\beta$ ) have been kept constant, while the other three $\left[E_{\mathrm{st}}, l_{\mathrm{sv}}\right.$ and $\left.Y_{0}(n)\left(\equiv Y_{n, 0}\right)\right]$ have been 
varied. The values of these five parameters have been specified by adopting the physicochemical properties of a reference case, that is, paracetamol crystallization from an ethanol solution at 20 ${ }^{\circ} \mathrm{C}$; the physicochemical properties and the corresponding values of the model parameters are reported in Tables 1 and 2, respectively.

\section{Table 1. Physicochemical Properties of the Paracetamol-} Ethanol System at $20^{\circ} \mathrm{C}$

\begin{tabular}{lll}
\multicolumn{1}{c}{ properties } & \multicolumn{1}{c}{ value } & \multicolumn{1}{c}{ description } \\
molecular weight of the solute, $M_{\mathrm{w}}$ & $0.151 \mathrm{~kg} \mathrm{~mol}^{-1}$ & \\
solid density, $\rho_{\mathrm{s}}$ & $1263 \mathrm{~kg} \mathrm{~m}^{-3}$ & \\
solvent density, $\rho_{\text {solv }}$ & $789 \mathrm{~kg} \mathrm{~m}^{-3}$ & \\
mass-based bulk solubility, $\bar{c}_{\mathrm{e}}$ & $0.180 \mathrm{~kg} \mathrm{~kg}^{-1}$ & ${\text { from ref } 28{ }^{a}}$ \\
bulk solubility, $c_{\mathrm{e}}$ & $5.65 \times 10^{26} \mathrm{~m}^{-3}$ & $\equiv \bar{c}_{\mathrm{e}} N_{\mathrm{A}} \rho_{\text {solv }} / M_{\mathrm{w}}{ }^{b}$ \\
specific surface energy, $\gamma$ & $0.0111 \mathrm{~J} \mathrm{~m}^{-2}$ & assumed value. \\
molecular volume of the solute, $V_{1}$ & $1.99 \times 10^{-28} \mathrm{~m}^{3}$ & $\equiv M_{\mathrm{w}} / \rho_{\mathrm{s}} N_{\mathrm{A}}{ }^{b}$ \\
molecular surface area of the solute, $b$ & $1.65 \times 10^{-18} \mathrm{~m}^{2}$ & $\equiv k_{\mathrm{s}}\left(V_{1} / k_{\mathrm{v}}\right)^{2 / 3 d}$ \\
monomer solubility, $C_{1, \mathrm{e}}$ & $4.82 \times 10^{26} \mathrm{~m}^{-3}$ & $\equiv C_{1}(0)$
\end{tabular}

${ }^{a}$ The solubility is based on solute mass per solvent mass. ${ }^{b} N_{\mathrm{A}}$ is the Avogadro number. ${ }^{c}$ The specific surface energy is within the range from an experimentally fitted value $\left(0.0043 \mathrm{~J} \mathrm{~m}^{-2}\right.$ at $40-46{ }^{\circ} \mathrm{C}$ by ref $29)$ to a theoretically predicted value $\left(0.0134 \mathrm{~J} \mathrm{~m}^{-2}\right.$ at $20^{\circ} \mathrm{C}$ by eq 12 in ref 26). ${ }^{d}$ Spherical shape is assumed: $k_{\mathrm{s}}=\pi$ and $k_{\mathrm{v}}=\pi / 6$.

The parameters $\beta$ and $\Omega$ have been calculated using eqs 25 and 26, respectively, with the physicochemical properties in Table $1: \beta=0.0958$ and $\Omega=4.5$. The parameter $E_{\text {st }}=\{1,1.1$, $1.2\}$ reflects the strength of interparticle interactions, and its values are conservative estimates of $E_{\mathrm{st}}$, ranging between 1.04 and 1.26 (see Section 3 in part I of this series ${ }^{27}$ ). The parameter $l_{\text {st }}$ represents the range of the stabilization effect. A wide range of $\{0,1,10,100\}$ has been investigated; a realistic value has been estimated to be around 4 in part I. ${ }^{27}$ By setting either $E_{\text {st }}=1$ (zero strength) or $l_{\text {st }}=0$ (zero thickness) or both, the stabilization effect can be eliminated, thus reducing the new SNIPE model to the Szilard model.

The last parameter $Y_{0}(n)$ determines the initial condition: the initial supersaturation $s_{0}$ via $Y_{0}(n=1)$, the initial subcritical and critical cluster concentrations via $Y_{0}\left(2 \leq n \leq n^{*}\right)$, and the initial seed size distribution via $Y_{0}\left(n>n^{*}\right)$. The initial supersaturation $s_{0}$ is equivalent to $Y_{0}(n=1)$ (see eqs 7 and $24 \mathrm{~b}$ ), and the studied range is $s_{0}=1.52-2.34$. For the subcritical and critical clusters, it is typical to assume that they are initially absent, ${ }^{21,22}$ thus setting $Y_{0}\left(2 \leq n \leq n^{*}\right)=0$. To define the initial seed crystal distribution $Y_{0}\left(n>n^{*}\right)$, it is required to set the initial amount of seeds and the initial probability density function (PDF) of the crystal size. The initial amount of seeds has been kept constant by fixing the initial solid volume fraction $\phi_{\mathrm{s}, 0}=0.0016$. This condition corresponds to $1 \mathrm{~g}$ of seeds in a $500 \mathrm{~mL}$ solution, a reported experimental condition that enables secondary nucleation in the paracetamol-ethanol system at $20{ }^{\circ} \mathrm{C} .{ }^{30}$ For the initial PDF, two cases are considered. In Sections 4.1 and 4.2, we use a narrow Gaussian distribution (characterized by the mean size 50 $\mu \mathrm{m}$ and the standard deviation $0.5 \mu \mathrm{m}$ ) to discuss the general behavior of the developed model. In Section 4.3, to compare our simulation results with experimental observations qualitatively, we use a log-normal distribution that adequately characterizes an experimental seed size distribution (explained in detail in Section 4.3).

The simulation results are presented in three parts. In Section 4.1 , we demonstrate the key features of the model in describing the time evolution of a cluster size distribution, including the formation of primary and secondary nuclei, thus leading to a bimodal crystal size distribution. Moreover, we verify that the stabilization effect can indeed cause secondary nucleation. In Section 4.2 , we present the results of a sensitivity analysis that elucidates the effect of the simulation parameters on the final crystal size distribution. Finally, in Section 4.3, we make a qualitative comparison between simulation results obtained using the SNIPE model and experimental observations reported in the literature.

4.1. Demonstration of the Stabilization Effect. The developed model can provide a comprehensive description of batch crystallization processes in which the growth of seeds can occur together with secondary nucleation induced by the interparticle energies. These processes are simulated in the model such that the initial cluster size distribution evolves over time with or without the stabilization effect while experiencing a series of molecule attachments (i.e., growth) and detachments (i.e., dissolution). The model yields the time evolution of a cluster size distribution $Y_{n}(\tau)$ that reveals insightful dynamics of the three key populations in the crystallization processes, namely, the populations of subcritical clusters, nuclei, and seed crystals. We emphasize that this type of insightful information cannot be attained using a (classical) population balance model. $^{21}$

In this section, we demonstrate how the stabilization effect can cause secondary nucleation by examining three simulation results. Two results are obtained from the simulations with no stabilization effect (i.e., $E_{\text {st }}=1$ and $l_{\text {st }}=0$ ) at two levels of the initial supersaturation $s_{0}$ : high level $s_{0}=2.22$ (Figure 1a) and low level $s_{0}=1.93$ (Figure $1 \mathrm{~b}$ ). The other simulation result is obtained when including the stabilization effect (corresponding to $E_{\mathrm{st}}=1.2$ and $l_{\mathrm{st}}=10$ ) and at the low supersaturation $s_{0}=1.93$ (Figure 1c). All three simulations are illustrated in Figure 1 in terms of the volume-weighted size distributions $n Y_{n}$ (solid lines)

\section{Table 2. Model Parameters Used in the Simulations}

\begin{tabular}{|c|c|c|c|c|}
\hline symbol & & & value & description \\
\hline$\beta$ & 0.0958 & & & monomer volume fraction at phase equilibrium \\
\hline$\Omega$ & 4.5 & & & nondimensional surface energy \\
\hline$E_{\text {st }}$ & $1,1.1,1.2$ & & & strength of the stabilization effect \\
\hline$l_{\text {st }}$ & $0,1,10,100$ & & & effective range of the stabilization effect \\
\hline$Y_{0}(n)$ & $n=1$ & $1.52-2.34$ & & initial supersaturation $s_{0}$ \\
\hline & $2 \leq n \leq n^{*}$ & 0 & & initial subcritical and critical clusters \\
\hline & $n^{*}<n$ & $\begin{array}{l}\phi_{\mathrm{s}, 0} \\
q_{3}(L)^{a}\end{array}$ & $\begin{array}{l}0.0016 \\
\operatorname{normal}(50,0.5) \text { or } \log -\operatorname{normal}(5.14,0.51)\end{array}$ & $\begin{array}{l}\text { initial solid volume fraction } \\
\text { normal dist. in Sections } 4.1 \text { and } 4.2 \text {; log-normal dist. in Section } 4.3\end{array}$ \\
\hline
\end{tabular}

${ }^{a}$ Volume-weighted probability density function of the crystal size in micrometer $L(n)=10^{-6} d_{1} \sqrt[3]{n}$. 


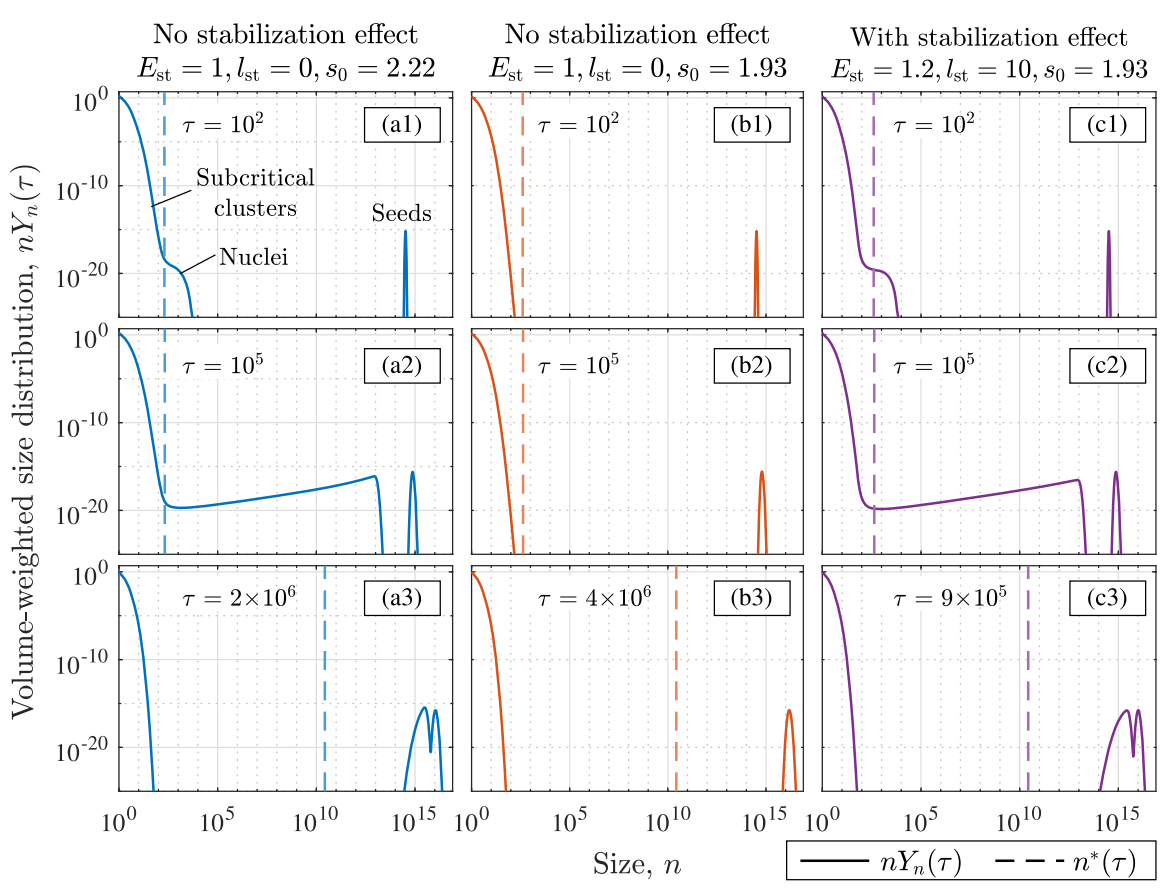

Figure 1. Comparison of three simulations conducted with two levels of the initial supersaturation $s_{0}$ and in the absence (a,b) or the presence (c) of the stabilization effect. For each simulation, the volume-weighted size distributions $\left(n Y_{n}(\tau)\right.$ vs $\left.n\right)$ at three times $\tau$ are depicted by the solid lines, and the corresponding critical sizes $\left(n^{*}(\tau)\right)$ are depicted by the dashed lines. In all relevant subplots, the point where the solid line intersects the dashed vertical line corresponds to the critical clusters, and its vertical coordinate is an indication of their concentration. A set of parameters for each simulation is as follows: (a) $E_{\mathrm{st}}=1 ; l_{\mathrm{st}}=0 ; s_{0}=2.22$. (b) $E_{\mathrm{st}}=1 ; l_{\mathrm{st}}=0 ; s_{0}=1.93$. (c) $E_{\mathrm{st}}=1.2 ; l_{\mathrm{st}}=10 ; s_{0}=1.93$.

and the critical sizes $n^{*}$ (dashed lines) at three different times. Note that $n Y_{n}$ is called volume-weighted distribution in the literature, ${ }^{21}$ though the distribution is weighed by the number of molecules because it is assumed quite rightly that the volume of a $n$-sized cluster is proportional to the number of molecules that comprise the cluster. ${ }^{19}$

The simulation without the stabilization effect at high supersaturation is analyzed first. The earliest snapshot [Figure 1(a1)] reveals the fast dynamics of a subcritical cluster population; a large number of subcritical clusters form rapidly. A small part of this population grows larger than the critical size, thus forming a population of primary nuclei through homogeneous nucleation. On the right-hand side of the figure, one can observe a seed crystal population in the form of a narrow Gaussian distribution. In the next snapshot [Figure 1(a2)], a growing nucleus population dramatically changes the cluster size distribution by increasing both the number and size of the nuclei. The third snapshot [Figure 1(a3)] is mainly characterized by a bimodal crystal size distribution consisting of both the seeds and the population of primary nuclei. All snapshots at the bottom row of Figure 1 represent the results at the final process times $\tau_{\mathrm{f}}$ at which the supersaturation is close to unity and the number of crystals is at its maximum (see Section S2 in the Supporting Information for more details).

The next simulation, illustrated in Figure $1 \mathrm{~b}$, is performed with no stabilization effect and a low level of the initial supersaturation $s_{0}=1.93$. In this simulation, a nucleus population does not form (see Figure $1(\mathrm{~b} 1, \mathrm{~b} 2)$ ); consequently, the seed crystals completely dominate the crystallization process through their growth, thus resulting in a unimodal crystal size distribution, as presented in Figure 1(b3). A comparison of the two simulations discussed so far (Figure la,b) indicates that the low initial supersaturation $s_{0}=1.93$ is insufficient to trigger primary nucleation in the bulk solution for the system under consideration here.The third simulation is illustrated in Figure 1c. Although this simulation begins with the low initial supersaturation, a substantial amount of nuclei appear [see Figure $1(\mathrm{c} 1, \mathrm{c} 2)]$ because of the stabilization effect. This stabilization effect enhances the overall driving force for crystallization and thus increases the concentration of the critical clusters by several orders of magnitude, as shown in Figure $1(\mathrm{c} 1, \mathrm{c} 2)$. Their subsequent growth and that of the seeds yield a bimodal crystal size distribution [see Figure 1(c3)]. The observed nucleation can be regarded as secondary nucleation caused by the stabilization effect, in other words, SNIPE. This result, obtained using the SNIPE model developed here, can explain why secondary nucleation can occur even when the initial supersaturation is at such a low level that primary nucleation does not occur.

4.2. Sensitivity Analysis. A sensitivity analysis has been conducted in two steps. First, various simulations have been carried out by altering three model parameters: the intensity of the stabilization effect $E_{\mathrm{st}}=\{1,1.1,1.2\}$, the effective range of the stabilization effect $l_{\text {st }}=\{0,1,10,100\}$, and the initial supersaturation $s_{0}=\{1.52,1.64,1.76,1.87,1.93,1.99,2.11$, $2.17,2.22,2.34\}$. Second, simulation results have been compared with respect to two characteristic quantities: the degree of nucleation, $\psi_{\mathrm{N}}$, which indicates how many new crystals have formed with respect to their initial count, and the degree of growth, $\psi_{\mathrm{G}}$, which specifies how much seed crystals have grown with respect to their initial size.

4.2.1. Degree of Nucleation and Growth. These two characteristic quantities can be readily calculated from a cluster size distribution, $Y_{n}(\tau)$, by using its moments. Hence, we first express the $j$-th moment $m_{j}(p, \tau)$ of the distribution $Y_{n}(\tau)$ in which the considered cluster size $n$ is larger than a given size $p$ 


$$
m_{j}(p, \tau)=\sum_{n=\lceil p\rceil}^{n_{\max }} n^{j} Y_{n}(\tau)
$$

Based on this definition, the degree of nucleation $\psi_{\mathrm{N}}$ can be defined by dividing the zeroth moment of the final crystal size distribution, $m_{0, f}^{*}$, by the initial one, $m_{0, \mathrm{i}}^{*}$

$$
\psi_{\mathrm{N}}=\frac{m_{0, \mathrm{f}}^{*}}{m_{0, i}^{*}}=\frac{m_{0}\left(n^{*}\left(\tau_{\mathrm{f}}\right), \tau_{\mathrm{f}}\right)}{m_{0}\left(n^{*}(0), 0\right)}
$$

where $n^{*}(\tau)$ is the critical size at time $\tau$. This equation gives $\psi_{\mathrm{N}}$ $\approx 1$ when nucleation is negligible, simply because the final number of crystals is close to the initial one (i.e., $m_{0, \mathrm{f}}^{*} \approx m_{0, \mathrm{i}}^{*}$ ). On the contrary, this definition yields $\psi_{\mathrm{N}} \gg 1$ when nucleation occurs because the final number of crystals is much larger than the initial one (i.e., $m_{0, \mathrm{f}}^{*} \gg m_{0, \mathrm{i}}^{*}$ ). Similarly, the degree of growth $\psi_{\mathrm{G}}$ can be defined by dividing the final volume-weighted average size of the seed crystals, $\bar{n}_{\text {seed,f }}$ by the initial one, $\bar{n}_{\text {seed,i }}$

$$
\psi_{\mathrm{G}}=\frac{\bar{n}_{\text {seed,f }}}{\bar{n}_{\text {seed,i }}}=\frac{\frac{m_{1}\left(n_{\text {seed,min }}\left(\tau_{\mathrm{f}}\right), \tau_{\mathrm{f}}\right)}{m_{0}\left(n_{\text {seed,min }}\left(\tau_{\mathrm{f}}\right), \tau_{\mathrm{f}}\right)}}{\frac{m_{1}\left(n_{\text {seed,min }}(0), 0\right)}{m_{0}\left(n_{\text {seed, } \min }(0), 0\right)}}
$$

where $n_{\text {seed,min }}(\tau)$ is the smallest size of a seed crystal size distribution at time $\tau$. This equation gives $\psi_{\mathrm{G}} \approx 1$ when the growth of seed crystals is negligible $\left(\bar{n}_{\text {seed,f }} \approx \bar{n}_{\text {seed,i }}\right)$, whereas it gives $\psi_{\mathrm{G}}>1$ when the seeds grow noticeably $\left(\bar{n}_{\text {seed, } \mathrm{f}}>\bar{n}_{\text {seed,i }}\right)$.

4.2.2. Results. The effect of the initial supersaturation $s_{0}$ on $\psi_{\mathrm{N}}$ and $\psi_{\mathrm{G}}$ in the absence of the stabilization effect $\left(E_{\mathrm{st}}=1\right.$ and $l_{\mathrm{st}}$ $=10)$ is illustrated in Figure 2 by the circle markers. When the initial supersaturation $s_{0}$ is below the threshold value $s_{\mathrm{th}}\left(E_{\mathrm{st}}=1\right)$ $=2.17$ (the left side of the vertical dotted line in Figure 2), $\psi_{\mathrm{N}}$ remains around 1 (see Figure $2 \mathrm{a}$ ), while $\psi_{\mathrm{G}}$ increases linearly with $s_{0}$ (see Figure $2 \mathrm{~b}$ ). By increasing $s_{0}$ beyond the threshold value $s_{\mathrm{th}}\left(E_{\mathrm{st}}=1\right), \psi_{\mathrm{N}}$ increases by orders of magnitude, whereas $\psi_{\mathrm{G}}$ drops to 1 . This effect of $s_{0}$ on $\psi_{\mathrm{N}}$ and $\psi_{\mathrm{G}}$ is also present in the simulations with the stabilization effect $\left(E_{\mathrm{st}}=\{1.1,1.2\}\right)$ but with the lower threshold values, $s_{\mathrm{th}}\left(E_{\mathrm{st}}=1.1\right)=2.10$ and $s_{\mathrm{th}}\left(E_{\mathrm{st}}=\right.$ $1.2)=1.87$, as illustrated in Figure 2 by the square markers for $E_{\mathrm{st}}$ $=1.1$ and the triangle markers for $E_{\mathrm{st}}=1.2$. In all three cases, if $s_{0}$ $<s_{\mathrm{th}}$, the growth of the seeds is the only dominant crystallization mechanism, indicated by $\psi_{\mathrm{N}} \approx 1$ and $\psi_{\mathrm{G}}>1$. By increasing $s_{0}$ beyond $s_{\mathrm{th}}$, the dominant crystallization mechanism continuously shifts from growth to primary nucleation (in the case of $E_{\text {st }}$ $=1$ ) or secondary nucleation (in the case of $E_{\mathrm{st}}>1$ ), indicated by $\psi_{\mathrm{N}} \gg 1$ and $\psi_{\mathrm{G}} \rightarrow 1$. Furthermore, the threshold initial supersaturation $s_{\text {th }}$ decreases with increasing strength of the stabilization effect $E_{\mathrm{st}}$. This suggests that secondary nucleation by interparticle energies can occur with a smaller value of $s_{0}$ if a system exhibits strong interparticle interactions between seeds and molecular clusters.

Next, we shift our attention to another group of simulations for studying the influence of $s_{0}$ and $l_{\text {st }}=\{1,10,100\}$ at a fixed value of $E_{\mathrm{st}}=1.2$. In the following, the degree of nucleation $\psi_{\mathrm{N}}$ alone is sufficient to demonstrate the impact of the model parameters. A higher value of $l_{\text {st }}$ means a longer effective range of the interparticle energies. As illustrated in Figure 3, although we increase $l_{\text {st }}$ by 1 or 2 orders of magnitude, the threshold supersaturation barely decreases. This minor effect of $l_{\text {st }}$ on the degree of nucleation $\psi_{\text {st }}$ sharply contrasts with the major effect of $E_{\text {st }}$.

To further understand the relative importance of these two parameters, a set of simulations have been executed with varying

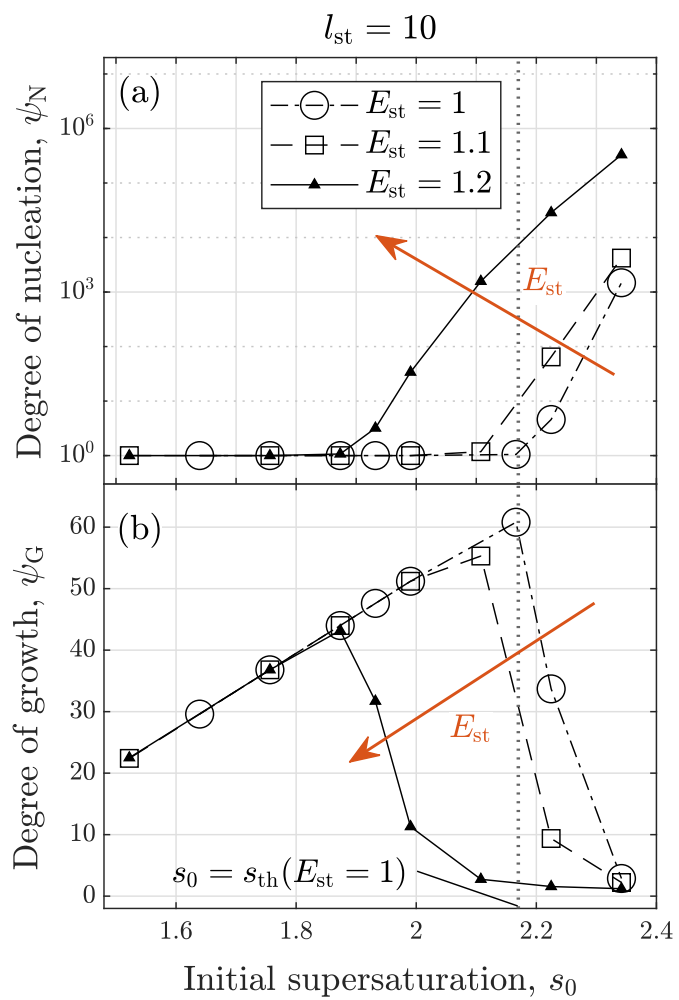

Figure 2. Degree of nucleation $\psi_{\mathrm{N}}$ is shown in plot (a) and degree of growth $\psi_{\mathrm{G}}$ is presented in plot (b) corresponding to the simulations conducted with varying values of the initial supersaturation $s_{0}$ and the stabilization effect strength $E_{\mathrm{st}}$ and a fixed value of the stabilization effect range $l_{\mathrm{st}}=10$. A vertical dotted line indicates the threshold initial supersaturation for $E_{\mathrm{st}}=1: s_{\mathrm{th}}\left(E_{\mathrm{st}}=1\right)$. Other lines are just a guide for the eye.

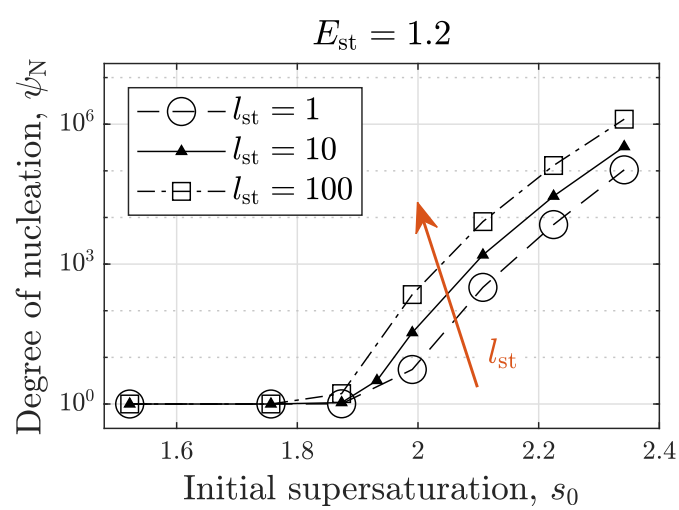

Figure 3. Degree of nucleation $\psi_{\mathrm{N}}$ of the simulations conducted with varying values of the initial supersaturation $s_{0}$ and the stabilization effect range $l_{\mathrm{st}}$ and a fixed value of the stabilization effect strength $E_{\mathrm{st}}=1.2$. Lines are just a guide for the eye.

values of $E_{\mathrm{st}}$ and $l_{\mathrm{st}}$ at a constant value of $s_{0}=2.22$, as illustrated in Figure 4. This figure clearly shows that the degree of nucleation $\psi_{\mathrm{N}}$ changes more dramatically when we change $E_{\mathrm{st}}$ than when $l_{\mathrm{st}}$ is varied. This result can be explained by considering the functional form of eqs $15 \mathrm{~b}$ and 23 , where $E_{\text {st }}$ determines the effective detachment rate constant $k^{\mathrm{d}, \text { eff }}$ more dominantly than $l_{\text {st }}$ does.

4.3. Comparison with Experimental Observations: Paracetamol Crystallization from an Ethanol Solution. In this section, we show the relevance of SNIPE-based simulations to experimental crystallization processes by 


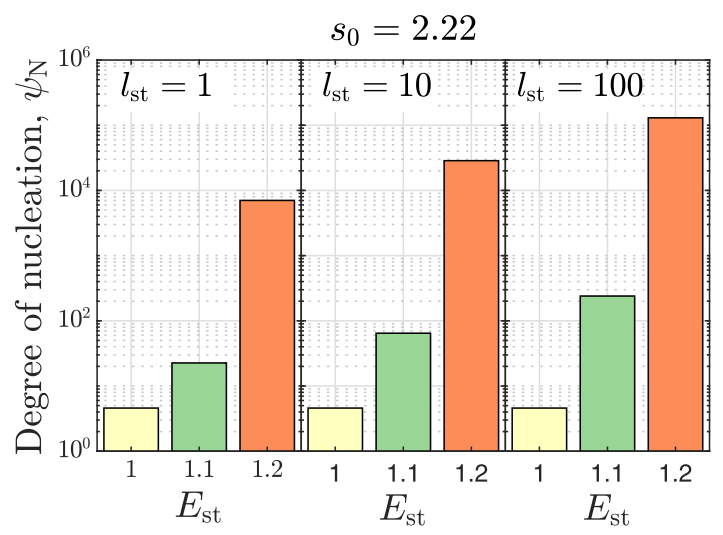

Figure 4. Degree of nucleation $\psi_{\mathrm{N}}$ of the simulations conducted with varying values of the stabilization effect strength $E_{\mathrm{st}}$ and the stabilization effect range $l_{\mathrm{st}}$ and a fixed value of the initial supersaturation $s_{0}=2.22$.

comparing our simulation results with available experimental observations qualitatively. For the comparison, simulation results [i.e., $\left.Y_{n}(\tau)\right]$ were converted into experimentally relevant quantities, namely, the bulk supersaturation $S$ and the volumeweighted PDF of the crystal size $q_{3}(L)$, as explained in Appendix $A$ and Appendix D, respectively. The conditions of the reference experiments were set up in the model as detailed in the following.

4.3.1. Setting up Experimental Conditions in the Model. References 30 and 31 are selected as benchmark experimental studies for two main reasons. One reason is that these experiments were conducted with the paracetamol-ethanol system, a widely studied system from various perspectives. $^{25,28,30-34}$ Another reason is that the chosen works were carried out systematically as a series; prior to their investigation of secondary nucleation, ${ }^{30}$ the authors extensively studied primary nucleation ${ }^{33,34}$ and growth ${ }^{31}$ for the same system, thus offering various types of experimental data sets obtained by the same researchers in a consistent manner.

In the following, we summarize the experimental conditions in refs 30 and 31 and explain how these conditions are reflected in the model parameters summarized in Table 2 . The reference experiments consist of seeded batch crystallization of paracetamol from an ethanol solution at $20{ }^{\circ} \mathrm{C}$; these conditions were implemented in the model by setting $\beta=0.0958$ and $\Omega=$ 4.5 , calculated using eqs 25 and 26, respectively, with the properties reported in Table 1 . The experiments are started by seeding $1 \mathrm{~g}$ of crystals in a $500 \mathrm{~mL}$ supersaturated solution; the corresponding seed size distribution is shown in Figure 5 in terms of the volume-weighted PDF $q_{3}(L)$ of the crystal size $L$; these conditions were reproduced in the model by setting the initial solid volume fraction $\phi_{\mathrm{s}, 0}=0.0016$ and by approximating the experimental seed size distribution with a log-normal distribution (see the dash-dotted line in Figure 5), characterized by the mean size 5.14 and standard deviation 0.51 of $\ln L$.

4.3.2. Comparison. Two sets of simulations were performed, namely, either in the absence or presence of the stabilization effect $\left(E_{\mathrm{st}}=1\right.$ and $l_{\mathrm{st}}=0$ and $E_{\mathrm{st}}=1.2$ and $l_{\mathrm{st}}=10$, respectively), each for estimating a threshold initial bulk supersaturation for primary nucleation and secondary nucleation, respectively. These estimates were compared with the values inferred from the experiments.

The results of the first set are presented in Figure 6a in terms of $q_{3}(L)$. When $S_{0}$ is larger than around 1.8 , the simulation yields a bimodal size distribution, thus indicating the occurrence of

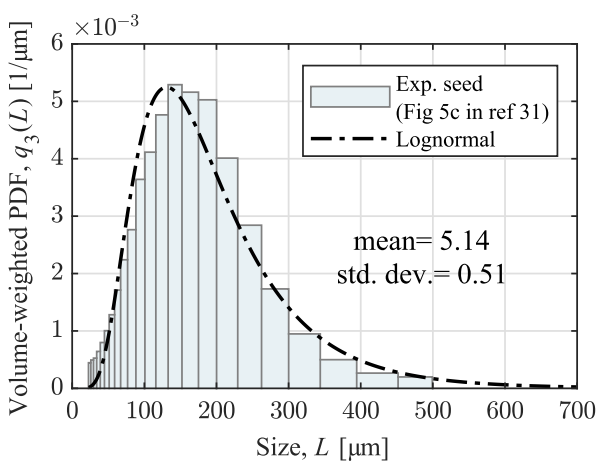

Figure 5. Experimental data of the volume-weighted PDF of the initial crystal size $\left(q_{3}(L)\right.$ vs $\left.L\right)$ shown by a bar graph and a fitted log-normal distribution (characterized by the mean 5.14 and standard deviation 0.51 of $\ln L$ ) by a dash-dotted line. The displayed experimental data are adapted with permission from Figure $5 \mathrm{c}$ in ref 31 . Copyright Elsevier (2011).

primary nucleation. This suggests that the threshold initial bulk supersaturation is around $S_{\mathrm{th}}\left(E_{\mathrm{st}}=1\right) \approx 1.8$. It is important to note that $S_{\mathrm{th}}\left(E_{\mathrm{st}}=1\right)$ varied around $\pm 5 \%$ when the specific surface energy $\gamma$ in Table 1 was varied around $\pm 10 \%$. A corresponding experimental value for the threshold supersaturation can be inferred from the experimental data in ref 31 that characterized the metastable zone width, reproduced in Figure $6 \mathrm{c}$. This figure shows that, at $20^{\circ} \mathrm{C}$, primary nucleation was detected when the initial bulk supersaturation was larger than 1.84 (corresponding to the point B in the figure), which is close to the value estimated by the model. This comparison demonstrates that the model can estimate a threshold supersaturation for primary nucleation adequately.

The outcome of the second simulation set is illustrated in Figure $6 \mathrm{~b}$, indicating that the threshold supersaturation for secondary nucleation falls in the range between 1.5 and 1.6. This range agrees well with the experimental observations in ref 30 where a value of the initial supersaturation in the range $S_{0}=$ 1.42-1.71 indeed caused secondary nucleation. This again shows that the developed model could be used to support future experiments, for instance, when selecting the initial supersaturation for a crystallization process.

We do acknowledge that the final PDFs in our simulations may not quantitatively match the experimental ones because of two main reasons. One reason is that, in experiments, secondary nucleation is caused by a combination of multiple mechanisms (e.g., attrition and SNIPE), but the developed model considers only the SNIPE mechanism. The second reason is that the final PDF can be critically modified by other crystallization phenomena (e.g., crystal breakage ${ }^{35-37}$ and agglomeration ${ }^{38,39}$ ), and these phenomena are not always predictable. In the reference experiment (ref 30), agglomeration clearly occurred, as evident from the reported SEM image of the final crystal population (see Figure 6d). Since the developed model describes neither agglomeration nor breakage, the simulation outcome cannot quantitatively agree with the experiments where any of these phenomena may occur. Though modeling crystal agglomeration and/or crystal breakage in a KRE model is possible, this is nevertheless beyond the scope of this work.

\section{CONCLUSIONS}

We have developed a mathematical model that simultaneously describes growth, homogeneous nucleation, and secondary nucleation caused by the interparticle energies, that is, due to the 

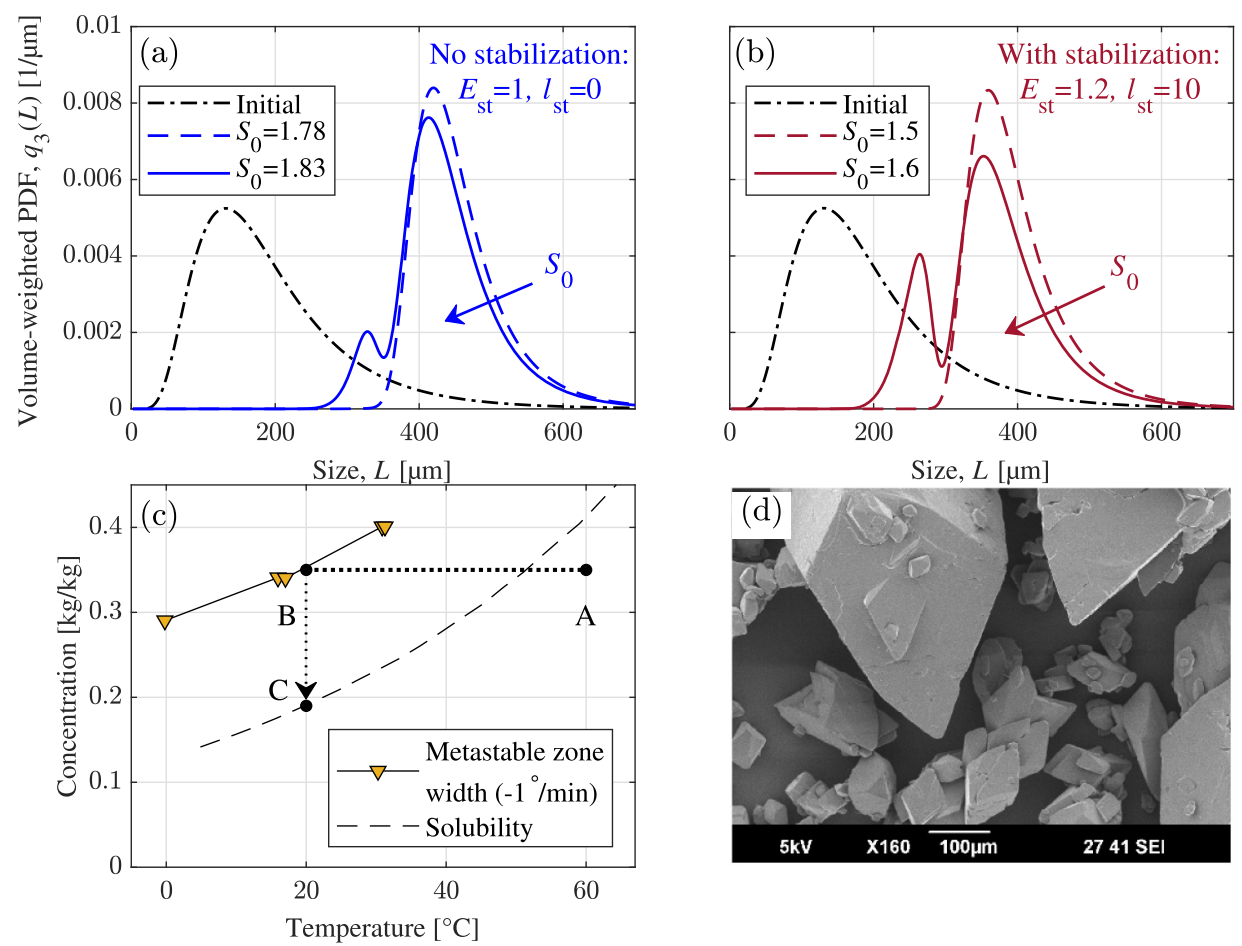

Figure 6. (a) Simulations conducted with no stabilization effect $\left(E_{\mathrm{st}}=1\right.$ and $\left.l_{\mathrm{st}}=0\right)$ at two values of the initial bulk supersaturation $S_{0}=\{1.78,1.83\}$. (b) Simulations performed with the stabilization effect (given by $E_{\mathrm{st}}=1.2$ and $l_{\mathrm{st}}=10$ ) at two values of the initial bulk supersaturation $S_{0}=\{1.5,1.6\}$. (c) Solubility and metastable zone width data. [Reproduced with permission from ref 31. Copyright Elsevier (2011)]. (d) SEM image of the final crystals showing agglomerates. [Reprinted with permission from ref 30. Copyright Elsevier (2012.)].

stabilization effect caused by the interactions between seeds and clusters in suspension. The key feature of the model is the incorporation of the stabilization effect into the conventional KRE model of nucleation. By simulating seeded batch crystallization of paracetamol from an ethanol solution at 20 ${ }^{\circ} \mathrm{C}$, this work demonstrates how the stabilization effect causes secondary nucleation at a low initial supersaturation by increasing the concentration of the critical clusters by orders of magnitude. Moreover, a sensitivity analysis has been carried out to understand the relative importance of three key simulation parameters: the initial supersaturation, $s_{0}$, the strength of the stabilization effect, $E_{\mathrm{st}}$ and the effective range thereof, $l_{\text {st }}$. The analysis shows that primary and secondary nucleation are enhanced to varying degrees on increasing any of the three parameters, and the parameter $E_{\text {st }}$ has a major effect on the stabilization effect, while the parameter $l_{\text {st }}$ has a minor effect. The simulation results are compared with the experimental observations in the literature qualitatively. The comparison verified a capability of the model to estimate a threshold initial bulk supersaturation at which primary or secondary nucleation occurs as the estimated values adequately agree with the values inferred from the reference experiments.

The model can be employed to obtain qualitative information on threshold supersaturation for nucleation, which is a useful tool for designing crystallization processes. Moreover, the developed model presents a new perspective on secondary nucleation mechanisms through which one can rationalize some experimental observations that cannot be explained by the attrition mechanism, for instance, the formation of secondary nuclei whose polymorphic form or chiral structure is different from that of a seed, ${ }^{14-17}$ the occurrence of secondary nucleation caused by a seed crystal that was held stationary, ${ }^{11-13}$ and the appearance of secondary nuclei in a no-impeller crystallizer (e.g., airlift crystallizer ${ }^{40,41}$ ).

Finally, demonstrating that the theory developed in part I and II of this series has potential for application and experimental validation remains a challenge. To address this, the complex KRE model used here needs to be transformed into a nucleation rate model, which can be used in a standard population balance modeling framework. This would prove that the SNIPE theory can describe experiments and would allow for a comparison of the SNIPE rate model with other well-recognized secondary nucleation models. These aspects will be addressed in part III of this series, ${ }^{42}$ where the practical relevance of secondary nucleation rate by interparticle energies will be proved.

\section{APPENDIX A}

\section{Mass Balance in Liquid Phase}

The solute concentration $c(\Delta \mu)$ at a given $\Delta \mu$ is equal to the total number of the solute molecules forming subcritical and critical clusters, which can be written as

$$
c=\sum_{n=1}^{n^{*}} n Z_{n}
$$

where the critical size $n^{*}(\Delta \mu)$ is defined according to the $\mathrm{CNT}^{19}$

$$
n^{*}=\left(\frac{2 \gamma b}{3 \Delta \mu}\right)^{3}
$$

Equation A.1 was applied to calculate the bulk supersaturation $S=c / c_{\mathrm{e}}$, which was used only for comparing simulation results with the relevant experimental observations in Section 4.3. Note that the monomer-based supersaturation $s$ was used to define the driving force $\Delta \mu$ (see eq 7). At phase equilibrium (i.e., $\Delta \mu=0)$, 
$Z_{n}$ can be approximated by the equilibrium concentration $C_{n}$ (eq $6)$ and $c(0)$ is equal to the bulk solubility $c_{\mathrm{e}}$ by definition; eq A.1 leads to

$$
c_{\mathrm{e}}=\sum_{n=1}^{\infty} n C_{n} \approx \sum_{n=1}^{n_{\max }} n C_{n}
$$

Finally, $C_{0}$ can be obtained by substituting eqs 6 and 8 into eq A.3

$$
C_{0}=\frac{c_{\mathrm{e}}}{\sum_{n=1}^{n_{\max }} n \exp \left(-\gamma b n^{2 / 3} / k_{\mathrm{B}} T\right)}
$$

\section{APPENDIX B}

\section{Nondimensionalization}

Nondimensionalization of differential equations is a convenient technique that makes physical quantities in differential equations dimensionless through the chain rule and some algebra. This technique effectively decreases the number of model parameters by grouping them into a smaller set. ${ }^{43}$ This can ease computational burden significantly because one simulation result in a nondimensional model can represent multiple results in the original model. For nondimensionalization, two dimensionless variables $Y_{n}$ and $\tau$ (see eqs 24a and 24b) are introduced. Note that $Y_{1}$ is equivalent to $s$ by definition (see eq 7) and that the time $t$ is scaled by the characteristic time for monomer attachments at phase equilibrium, that is, $\left(k_{1}^{\mathrm{a}} C_{1, \mathrm{e}}\right)^{-1}$. Substituting these definitions into the governing equations (eqs $2 \mathrm{~b}$ and 22 ) leads to

$$
\begin{aligned}
\frac{\mathrm{d} Y_{n}}{\mathrm{~d} \tau} & =\eta_{n-1} Y_{1} Y_{n-1}+\xi_{n+1} Y_{n+1}-\eta_{n} Y_{1} Y_{n}-\xi_{n} Y_{n} \\
\quad(n & \left.=2,3, \ldots, n_{\max }\right) \\
\frac{\mathrm{d} Y_{1}}{\mathrm{~d} \tau} & =-\sum_{n=2}^{n_{\max }} n \frac{\mathrm{d} Y_{n}}{\mathrm{~d} \tau}
\end{aligned}
$$

where $\eta_{n}$ and $\xi_{n}(\tau)$ are the nondimensional rate constants defined as

$$
\begin{aligned}
\eta_{n}= & n^{2 / 3} \\
\xi_{n}= & {\left[\frac{\phi_{\mathrm{st}} E_{\mathrm{st}}^{n-1}+\phi_{\mathrm{b}}}{\phi_{\mathrm{st}} E_{\mathrm{st}}^{n}+\phi_{\mathrm{b}}}\right](n-1)^{2 / 3} } \\
& \exp \left(\Omega\left[n^{2 / 3}-(n-1)^{2 / 3}\right]\right)
\end{aligned}
$$

The corresponding initial condition is

$$
Y_{n}(0)=Y_{n, 0}
$$

It is worth noting that one can recast eqs 14 and $15 \mathrm{~b}$ using dimensionless quantities as

$$
\begin{aligned}
& \phi_{\mathrm{s}}=\beta \sum_{n=n^{*}+1}^{n_{\max }} n Y_{n} \\
& \phi_{\mathrm{st}}=6 \beta l_{\mathrm{st}} \sum_{n=n_{\mathrm{st}}}^{n_{\max }} n^{2 / 3} Y_{n}
\end{aligned}
$$

\section{APPENDIX C}

\section{Solution of the SNIPE Model}

The governing equations (eq A.5a) are a set of integro differential equations as the rate constants $\xi(\tau)$ depend on the moments of a cluster size distribution $Y(\tau)$. These equations can be approximated by a set of ordinary differential equations (ODEs) by treating $\xi$ as constants at every time integration, for example, for the $j$-th integration, $\xi_{n}(\tau)=\xi_{n}\left(\tau_{j}\right)$ for $\tau_{j} \leq \tau<\tau_{j+1}$. This approximation is reasonable because the cluster concentrations $Y_{n}$ vary much faster than $\xi_{n}$, yielding a small integration time step $\Delta \tau_{j} \equiv \tau_{j+1}-\tau_{j}$, and during each time integration, the relative change in $\xi_{n}$ is very small, satisfying the following condition

$$
\frac{\left.\frac{\partial \xi_{n}}{\partial \tau}\right|_{\tau_{j}} \Delta \tau_{j}}{\xi_{n}\left(\tau_{j}\right)} \ll 1
$$

Accordingly, we can approximate $\xi_{n}(\tau)$ by $\xi_{n}\left(\tau_{j}\right)$ for $\tau_{j} \leq \tau<$ $\tau_{j+1}$ as

$$
\begin{aligned}
& \xi_{n}(\tau) \approx \xi_{n}\left(\tau_{j}\right)+\left.\frac{\partial \xi_{n}}{\partial \tau}\right|_{\tau_{j}}\left(\tau-\tau_{j}\right) \\
& \approx \xi_{n}\left(\tau_{j}\right) \quad(\because \text { eq A. } 11)
\end{aligned}
$$

Moreover, the governing equations (eqs A.5a and A.5b) are a set of $n_{\max }$ discrete rate equations, each equation describing the concentration of clusters, with the size $n$ ranging from 1 to $n_{\max }$. For describing the dynamics of large crystals, for instance, 800 $\mu \mathrm{m}$ crystals, the required $n_{\max }$ can be on the order of $10^{18}$. This turns the governing equations into a set of $10^{18}$ or more differential equations that definitely causes formidable computational burden. This issue can be adequately resolved by approximating the discrete rate equations with the FokkerPlanck partial differential equation (FPE). ${ }^{21,22,44}$ The FPE can be readily derived by applying the second-order Taylor series expansion to the two terms of eq A.5a around n, namely, $\eta_{n-1} Y_{1} Y_{n-1}$ and $\xi_{n+1} Y_{n+1}$, ${ }^{19}$ yielding

$$
\begin{aligned}
& \frac{\partial \tilde{Y}}{\partial \tau}+\frac{\partial}{\partial \tilde{n}}\left(v \tilde{Y}-\frac{1}{2} \frac{\partial(H \tilde{Y})}{\partial \tilde{n}}\right)=0 \\
& v(\tilde{n})=\eta(\tilde{n}) Y_{1}-\xi(\tilde{n}) \\
& H(\tilde{n})=\eta(\tilde{n}) Y_{1}+\xi(\tilde{n})
\end{aligned}
$$

where $\tilde{Y}(\tilde{n}, \tau)$, the continuous counterpart of $Y_{n}$, is a function of the continuous size $\tilde{n}, v(\tilde{n}, \tau)$ is the growth rate (i.e., the net molecule attachment frequency) of the $n$-sized cluster, and $H(\tilde{n}, \tau)$ is the dispersion coefficient of the clusters along the size axis $\tilde{n}$, reflecting a fluctuating change in the cluster size $\tilde{n}{ }^{19}$ However, because the approximation with the FPE is not sufficiently accurate for small $\tilde{n}$, it is common to use the original discrete equations for small size (i.e., $n \leq N$ ) and the FPE for larger size (i.e., $\tilde{n}>N),{ }^{21,22,44}$ yielding a hybrid size coordinate. In this work, $N$ is set to 50 as it has been reported that increasing $N$ beyond 50 does not improve the accuracy of the solutions. ${ }^{21,22}$ To smoothly combine the FPE with the discrete equations, it is necessary to adapt eqs 27, A.5b, A.9, and A.10 to the hybrid size domain, spatially discretize the FPE by using a suitable discretization method, and impose two boundary conditions, as explained in Section S1 in the Supporting Information. In this work, the incorporation of the FPE reduces the total number of 
ODEs substantially from a value on the order of $10^{18}$ to 4000 . The discrete rate equations and the spatially discretized (or semi-discrete) FPE are integrated over time using the MATLAB's ode15s solver for stiff ODEs.

\section{APPENDIX D}

\section{Conversion of Simulation Results}

This section explains a procedure for converting a crystal size distribution $Y_{n>n^{*}}(\tau)$ to a corresponding volume-weighted PDF $q_{3}(L, \tau)$ of the crystal size in micrometer, $L$. The size variable $n$ is connected to the size $L$ through the expression for a cluster volume as

$$
n V_{1}=10^{-18} k_{\mathrm{v}} L^{3}
$$

where the number $10^{-18}$ converts the unit $\left[\mu \mathrm{m}^{3}\right]$ into $\left[\mathrm{m}^{3}\right]$. By rearranging eq A.17 to obtain $L$, we can derive a conversion function $L=f(n)$

$$
L=f(n)=10 \sqrt[6]{\frac{V_{1}}{k_{\mathrm{v}}} n}
$$

A crystal size distribution $Y_{n>n^{*}}(\tau)$ can be first converted to a volume-weighted PDF $\bar{q}_{1}\left(n>n^{*}, \tau\right)$ of the size $n$

$$
\bar{q}_{1}(n, \tau)=\frac{n Y_{n}(\tau)}{m_{1}\left(n^{*}, \tau\right)} \quad \text { for } \quad n>n^{*}
$$

Next, by using eq A.18 and applying the change-of-variable technique, $\tilde{q}_{1}(n, \tau)$ can be converted to $q_{3}(L, \tau)$

$$
q_{3}(L, \tau)=\frac{\bar{q}_{1}(n, \tau)}{\left|f^{\prime}(n)\right|}=3 \times 10^{-6} \sqrt[3]{\frac{k_{\mathrm{v}}}{V_{1}} n^{2}} \bar{q}_{1}(n, \tau)
$$

\section{ASSOCIATED CONTENT}

\section{SI Supporting Information}

The Supporting Information is available free of charge at https://pubs.acs.org/doi/10.1021/acs.cgd.1c00928.

Adaptation of eqs $24 \mathrm{~b}$, A.5b, A.9, and A.10 to the hybrid size domain; $\tilde{n}$-space discretization of the FPE; two boundary conditions to smoothly combine the FPE with the original discrete equations; numerical method to solve FPE; time evolution of supersaturation; and the zeroth moment of a crystal population (PDF)

\section{AUTHOR INFORMATION}

\section{Corresponding Authors}

Byeongho Ahn - Institute of Energy and Process Engineering, ETH Zurich, Zurich 8092, Switzerland; 으이이.org/00000002-4405-5041; Email: ahn@ipe.mavt.ethz.ch

Luca Bosetti - Institute of Energy and Process Engineering, ETH Zurich, Zurich 8092, Switzerland; 이이.org/00000001-6657-6510; Email: bosettil@ipe.mavt.ethz.ch

Marco Mazzotti - Institute of Energy and Process Engineering, ETH Zurich, Zurich 8092, Switzerland; 이이.org/00000002-4948-6705; Email: marco.mazzotti@ipe.mavt.ethz.ch

Complete contact information is available at:

https://pubs.acs.org/10.1021/acs.cgd.1c00928

\section{Notes}

The authors declare no competing financial interest.

\section{ACKNOWLEDGMENTS}

This project has received funding from the European Research Council (ERC) under the European Unions Horizon 2020 Research and Innovation Program under grant agreement no. 788607.

\section{NOMENCLATURE}

\section{Acronyms}

API, active pharmaceutical ingredient

SNIPE, secondary nucleation caused by interparticle energies ECSN, embryo coagulation secondary nucleation

CNT, classical nucleation theory

$\mathrm{KRE}$, kinetic rate equation

ODE, ordinary differential equation

FPE, Fokker-Planck partial differential equation

PDF, probability density function

\section{Greek symbols}

$\alpha$, sticking coefficient, $[-]$

$\beta$, volume fraction of the monomers at phase equilibrium, $[-]$

$\Delta \mu$, driving force for crystallization, [J]

$\eta$, non-dimensional attachment rate constant, $[-]$

$\gamma$, specific surface energy, $\left[\mathrm{Jm}^{-2}\right]$

$\Omega$, non-dimensional surface energy, $[-]$

$\phi$, volume fraction, $[-]$

$\psi$, degree of nucleation/growth, $[-]$

$\rho$, density, $\left[\mathrm{kg} \mathrm{m}^{-3}\right]$

$\tau$, non-dimensional time, $[-]$

$\xi$, non-dimensional detachment rate constant, $[-]$

Mathematical operators

$\because$, because

$\lceil$, least integer greater than or equal to

\section{Roman symbols}

$\bar{C}_{\mathrm{e}}$, mass-based bulk solubility, $\left[\mathrm{kg} \mathrm{kg}^{-1}\right]$

$\bar{n}$, volume-weighted average size, $[-]$

$\bar{q}_{1}$, volume-weighted probability density function of the cluster size $n,[-]$

$\bar{n}$, continuous cluster size in terms of the number of molecules, $[-]$

$\tilde{Y}$, non-dimensional concentration in the continuous size domain, $[-]$

$b$, surface area of a solute molecule, $\left[\mathrm{m}^{2}\right]$

$C$, cluster concentration at truly stable or metastable equilibrium, $\left[\mathrm{m}^{-3}\right]$

$c$, bulk solute concentration, $\left[\mathrm{m}^{-3}\right]$

$C_{0}$, concentration of nucleation sites, $\left[\mathrm{m}^{-3}\right]$

$D$, diffusion coefficient of the solute molecule in the solution, $\left[\mathrm{m}^{2} \mathrm{~s}^{-1}\right]$

$d$, characteristic length of a cluster, $[\mathrm{m}]$

$E_{\text {st }}$, strength of the stabilization effect, $[-]$

$f$, function converting $n$ to $L,[\mathrm{~m}]$

$H$, non-dimensional dispersion coefficient along the continuous size $\tilde{n},[-]$

$k_{\mathrm{B}}$, Boltzmann constant, $\left[\mathrm{JK}^{-1}\right]$

$k_{\mathrm{s}}$, shape factor for surface area, $[-]$

$k_{\mathrm{v}}$, shape factor for volume, $[-]$

$k^{\mathrm{a}}$, rate constant of molecule attachment, $\left[\mathrm{m}^{3} \mathrm{~s}^{-1}\right]$

$k^{\mathrm{d}}$, rate constant of molecule detachment, $\left[\mathrm{s}^{-1}\right]$

$L$, crystal size in micrometer, $[\mu \mathrm{m}]$

$l_{\mathrm{st}}$ effective range of the stabilization effect, $[-]$

$M_{\mathrm{w}}$, molecular weight, $\left[\mathrm{kg} \mathrm{mol}^{-1}\right]$

$m_{j}$, j-th moment of the distribution $Y_{n \geq p}(\tau),[-]$ 
$N$, upper bound of the discrete cluster size $n$ in the hybrid size coordinate, $[-]$

$n$, discrete cluster size in terms of the number of solute molecules, [-]

$N_{\mathrm{A}}$, Avogadro number, $\left[\mathrm{mol}^{-1}\right]$

$n_{\mathrm{st}}$ minimum cluster size to create the stabilization volume, $[-]$

$q_{3}$, volume-weighted probability density function of the crystal size $L,[\mu \mathrm{m}]$

$S$, bulk supersaturation (ratio), [-]

$s$, supersaturation (ratio) based on the monomer concentrations, $[-]$

$T$, absolute temperature, $[\mathrm{K}]$

$t$, time, $[\mathrm{s}]$

$V$, volume, $\left[\mathrm{m}^{3}\right]$

$v$, non-dimensional growth rate, $[-]$

$W$, cluster, [-]

$Y$, non-dimensional concentration, $[-]$

$Z$, cluster concentration, $\left[\mathrm{m}^{-3}\right]$

$\Delta G$, Gibbs free energy for forming a molecular cluster, [J]

\section{Superscripts and subscripts}

0 , initial value

$n$, $n$-sized clusters

*, critical nucleus

b, bulk solution

e, phase equilibrium

eff, effective

f, final

$\mathrm{G}$, growth

max, upper bound

min, lower bound

$\mathrm{N}$, nucleation

s, solid

seed, seed crystals

st, stabilization volume

susp, suspension

th, threshold

\section{REFERENCES}

(1) Agrawal, S. G.; Paterson, A. H. J. Secondary Nucleation: Mechanisms and Models. Chem. Eng. Commun. 2015, 202, 698-706.

(2) Bosetti, L.; Mazzotti, M. Population Balance Modeling of Growth and Secondary Nucleation by Attrition and Ripening. Cryst. Growth Des. 2020, 20, 307-319.

(3) Tyrrell, R.; De Souza, B.; Frawley, P. J. Particle Breakage: Limiting Conditions for Crystal-Crystallizer Collisions. Cryst. Growth Des. 2018, $18,617-622$.

(4) Xu, S.; Hou, Z.; Chuai, X.; Wang, Y. Overview of Secondary Nucleation: From Fundamentals to Application. Ind. Eng. Chem. Res. 2020, 59, 18335-18356.

(5) Zhang, D.; Wang, X.; Ulrich, J.; Tang, W.; Xu, S.; Li, Z.; Rohani, S.; Gong, J. Control of Crystal Properties in a Mixed-Suspension MixedProduct Removal Crystallizer: General Methods and the Effects of Secondary Nucleation. Cryst. Growth Des. 2019, 19, 3070-3084.

(6) Anwar, J.; Khan, S.; Lindfors, L. Secondary Crystal Nucleation: Nuclei Breeding Factory Uncovered. Angew. Chem., Int. Ed. 2015, 54, 14681-14684.

(7) de Souza, B.; Cogoni, G.; Tyrrell, R.; Frawley, P. J. Evidence of Crystal Nuclei Breeding in Laboratory Scale Seeded Batch Isothermal Crystallization Experiments. Cryst. Growth Des. 2016, 16, 3443-3453.

(8) Kondepudi, D. K.; Asakura, K. Chiral Autocatalysis, Spontaneous Symmetry Breaking, and Stochastic Behavior. Acc. Chem. Res. 2001, 34, 946-954.
(9) Buhse, T.; Durand, D.; Kondepudi, D.; Laudadio, J.; Spilker, S. Chiral Symmetry Breaking in Crystallization: The Role of Convection. Phys. Rev. Lett. 2000, 84, 4405-4408.

(10) Sathyamoorthy, S.; Moggridge, G. D.; Hounslow, M. J. Particle Formation during Anatase Precipitation of Seeded Titanyl Sulfate Solution. Cryst. Growth Des. 2001, 1, 123-129.

(11) Yousuf, M.; Frawley, P. J. Experimental Evaluation of Fluid Shear Stress Impact on Secondary Nucleation in a Solution Crystallization of Paracetamol. Cryst. Growth Des. 2018, 18, 6843-6852.

(12) Yousuf, M.; Frawley, P. J. Secondary Nucleation from Nuclei Breeding and Its Quantitative Link with Fluid Shear Stress in Mixing: A Potential Approach for Precise Scale-up in Industrial Crystallization. Org. Process Res. Dev. 2019, 23, 926-934.

(13) Yousuf, M.; Frawley, P. J. Quantitative Link between Secondary Nucleation and Mixing Hydrodynamics in Batch Cooling Crystallization: A New Approach in Process Development. Org. Process Res. Dev. 2019, 23, 2009-2019.

(14) Cui, Y.; Myerson, A. S. Experimental Evaluation of Contact Secondary Nucleation Mechanisms. Cryst. Growth Des. 2014, 14, $5152-5157$

(15) Qian, R.-Y.; Botsaris, G. D. Nuclei breeding from a chiral crystal seed of NaClO3. Chem. Eng. Sci. 1998, 53, 1745-1756.

(16) Denk, E. G.; Botsaris, G. D. Fundamental studies in secondary nucleation from solution. J. Cryst. Growth 1972, 13-14, 493-499.

(17) Callahan, C. J.; Ni, X.-W. An investigation into the effect of mixing on the secondary nucleation of sodium chlorate in a stirred tank and an oscillatory baffled crystallizer. CrystEngComm 2014, 16, 690697.

(18) Qian, R.-Y.; Botsaris, G. D. A new mechanism for nuclei formation in suspension crystallizers: the role of interparticle forces. Chem. Eng. Sci. 1997, 52, 3429-3440.

(19) Kashchiev, D. Nucleation; Elsevier, 2000.

(20) Farkas, L. Keimbildungsgeschwindigkeit in übersättigten Dämpfen. Z. Physiol. Chem. 1927, 125U, 236-242.

(21) Vetter, T.; Iggland, M.; Ochsenbein, D. R.; Hänseler, F. S.; Mazzotti, M. Modeling Nucleation, Growth, and Ostwald Ripening in Crystallization Processes: A Comparison between Population Balance and Kinetic Rate Equation. Cryst. Growth Des. 2013, 13, 4890-4905.

(22) Rempel, J. Y.; Bawendi, M. G.; Jensen, K. F. Insights into the Kinetics of Semiconductor Nanocrystal Nucleation and Growth. J. Am. Chem. Soc. 2009, 131, 4479-4489.

(23) Tahri, Y.; Kožíšek, Z.; Gagnière, E.; Chabanon, E.; Bounahmidi, T.; Mangin, D. Modeling the Competition between Polymorphic Phases: Highlights on the Effect of Ostwald Ripening. Cryst. Growth Des. 2016, 16, 5689-5697.

(24) Eder, C.; Choscz, C.; Müller, V.; Briesen, H. Jamininterferometer-setup for the determination of concentration and temperature dependent face-specific crystal growth rates from a single experiment. J. Cryst. Growth 2015, 426, 255-264.

(25) de Albuquerque, I.; Mazzotti, M.; Ochsenbein, D. R.; Morari, M. Effect of needle-like crystal shape on measured particle size distributions. AIChE J. 2016, 62, 2974-2985.

(26) Kashchiev, D.; van Rosmalen, G. M. Review: Nucleation in solutions revisited. Cryst. Res. Technol. 2003, 38, 555-574.

(27) Bosetti, L.; Ahn, B.; Mazzotti, M. Secondary Nucleation by Interparticle Energies. I. Thermodynamics. Cryst. Growth Des. 2021 DOI: 10.1021 /acs.cgd.1c00927

(28) Worlitschek, J.; Mazzotti, M. Model-based optimization of particle size distribution in batch-cooling crystallization of paracetamol. Cryst. Growth Des. 2004, 4, 891-903.

(29) Li, H.; Kawajiri, Y.; Grover, M. A.; Rousseau, R. W. Modeling of Nucleation and Growth Kinetics for Unseeded Batch Cooling Crystallization. Ind. Eng. Chem. Res. 2017, 56, 4060-4073.

(30) Frawley, P. J.; Mitchell, N. A.; Ó'Ciardhá, C. T.; Hutton, K. W. The effects of supersaturation, temperature, agitation and seed surface area on the secondary nucleation of paracetamol in ethanol solutions. Chem. Eng. Sci. 2012, 75, 183-197. 
(31) Mitchell, N. A.; Ó’Ciardhá, C. T.; Frawley, P. J. Estimation of the growth kinetics for the cooling crystallisation of paracetamol and ethanol solutions. J. Cryst. Growth 2011, 328, 39-49.

(32) Liu, Y.; Black, J. F. B.; Boon, K. F.; Cruz-Cabeza, A. J.; Davey, R. J.; Dowling, R. J.; George, N.; Hutchinson, A.; Montis, R. When Crystals Do Not Grow: The Growth Dead Zone. Cryst. Growth Des. 2019, 19, 4579-4587.

(33) Mitchell, N. A.; Frawley, P. J. Nucleation kinetics of paracetamolethanol solutions from metastable zone widths. J. Cryst. Growth 2010, 312, 2740-2746.

(34) Mitchell, N. A.; Frawley, P. J.; Ó’Ciardhá, C. T. Nucleation kinetics of paracetamol-ethanol solutions from induction time experiments using Lasentec FBRM. J. Cryst. Growth 2011, 321, 91-99.

(35) Salvatori, F.; Mazzotti, M. Manipulation of Particle Morphology by Crystallization, Milling, and Heating Cycles-A Mathematical Modeling Approach. Ind. Eng. Chem. Res. 2017, 56, 9188-9201.

(36) Salvatori, F.; Mazzotti, M. Experimental Characterization and Mathematical Modeling of Breakage of Needle-like Crystals in a Continuous Rotor-Stator Wet Mill. Cryst. Growth Des. 2018, 18, 59575972.

(37) Salvatori, F.; Mazzotti, M. Manipulation of Particle Morphology by Crystallization, Milling, and Heating Cycles: Experimental Characterization. Ind. Eng. Chem. Res. 2018, 57, 15522-15533.

(38) Lindenberg, C.; Schöll, J.; Vicum, L.; Mazzotti, M.; Brozio, J. 1Glutamic Acid Precipitation: Agglomeration Effects. Cryst. Growth Des. 2008, 8, 224-237.

(39) Orlewski, P. M.; Mazzotti, M. Modeling of Mixing-Precipitation Processes: Agglomeration. Chem. Eng. Technol. 2020, 43, 1029-1039.

(40) Anisi, F.; Kramer, H. J. M. Crystallization kinetics in an airlift and a stirred draft tube crystallizer; Secondary nucleation models revisited. Chem. Eng. Res. Des. 2018, 138, 200-211.

(41) Lakerveld, R.; van Krochten, J. J. H.; Kramer, H. J. M. An Air-Lift Crystallizer Can Suppress Secondary Nucleation at a Higher Supersaturation Compared to a Stirred Crystallizer. Cryst. Growth Des. 2014, $14,3264-3275$.

(42) Ahn, B.; Bosetti, L.; Mazzotti, M. Secondary Nucleation by Interparticle Energies. III. Nucleation Rate Model. Cryst. Growth Des. 2021, submitted.

(43) Langtangen, H. P.; Pedersen, G. K. Scaling Differ. Equations; Springer International Publishing: Cham, 2016.

(44) Ozkan, G.; Ortoleva, P. A mesoscopic model of nucleation and Ostwald ripening/stepping: Application to the silica polymorph system. J. Chem. Phys. 2000, 112, 10510-10525. 